\title{
Design of an indoor/outdoor seamless positioning and attitude detection system
}

\author{
Mingming Bian ${ }^{1, *}$ Qiang Guo ${ }^{1}$ Xiaona Sun ${ }^{2}$ Baoquan $\mathrm{Gu}^{1}$ Linlin Bian ${ }^{3}$ \\ ${ }^{1}$ Binzhou Polytechnic, Shandong,China \\ ${ }^{2}$ Shan Dong Heng Tai Group, Shandong,China \\ ${ }^{3}$ Shan Dong Befar Group, Shandong,China \\ *Corresponding author. Email:rentian9876@163.com
}

\begin{abstract}
Aiming at the problems such as inaccurate positioning caused by indoor blocking of satellite positioning signals and the demand of personnel attitude detection, this paper designs a seamless positioning and attitude detection system based on GPS and inertial navigation, the system uses STM32 as the main controller, GPS and iNEMO sensors collect positioning and attitude detection data, and the processed information is sent to the upper computer through GPRS module SIM300, it realizes remote indoor and outdoor seamless positioning and attitude detection functions.
\end{abstract}

Keywords: Positioning,Attitude detection,STM32

\section{INTRODUCTION}

Beidou, GPS, GLONASS and Galileo are the four major satellite positioning systems at present. Outdoor positioning has wide coverage and high accuracy, but in indoor and urban complex environment, they are easily affected by signal blocking and attenuation, resulting in inaccurate positioning results or even unable to locate. In response, indoor positioning technology has gradually developed, mainly including RFID technology, WIFI technology, Bluetooth technology, ultrasonic technology, inertial navigation technology, etc. Outdoor and indoor positioning technologies are independent of each other, but seamless docking from outdoor to indoor or from indoor to outdoor cannot be realized. Seamless positioning is very important for specific personnel. It is extremely important to know the position and posture information of the firemen in real time when the firemen enter the scene of the serious fire to rescue. In order to meet this demand, this paper designs a seamless positioning and attitude detection system based on GPS and inertial navigation technology.

\section{OVERALL SCHEME DESIGN}

This system positioning adopts the method of combining GPS and pedestrian track estimation positioning technology, among which pedestrian track estimation positioning adopts the method of measuring walking distance based on step frequency and step length. The system merges the data of the accelerator, magnetometer and gyroscope of the inertial module iNEMO, and obtains three attitude angles of yaw, pitching and rolling. After processing the three-axis acceleration data of the waist iNEMO, the step frequency detection is realized, and the step counting function is realized. After the step counting is accurate, the yaw angle information after the fusion of the waist iNEMO is utilized, and the step size model is combined, calculate and decompose pedestrian tracks and convert them into longitude and latitude information. Receiving longitude and latitude information through GPS module, and processing the error of the output data of GPS module, when the quality of GPS signal is good, the positioning result completely adopts GPS positioning information, and use GPS positioning information to 
correct the pedestrian track estimation information in real time; When the quality of GPS signal is average, combine GPS positioning information and pedestrian track estimation positioning information, and take the fused information as the final positioning result; When the quality of GPS signal is poor, the positioning result adopts pedestrian track to calculate the positioning information. The system uses two iNEMO sensors to realize attitude detection, and the two sensors are respectively installed on the legs and waist to detect the attitude angle. According to the physiological characteristics of human body movement, posture information can be obtained by analyzing the data output by iNEMO sensors in legs and waist.

The system is mainly composed of STM32 main control module, GPS module, iNEMO module, GPRS module, display module and power supply module, etc. The information collected by GPS module and inertial navigation iNEMO module is processed by STM32 main control module, and then sent to upper computer through GPRS module to complete positioning and attitude detection. The system framework is shown in Figure 1.

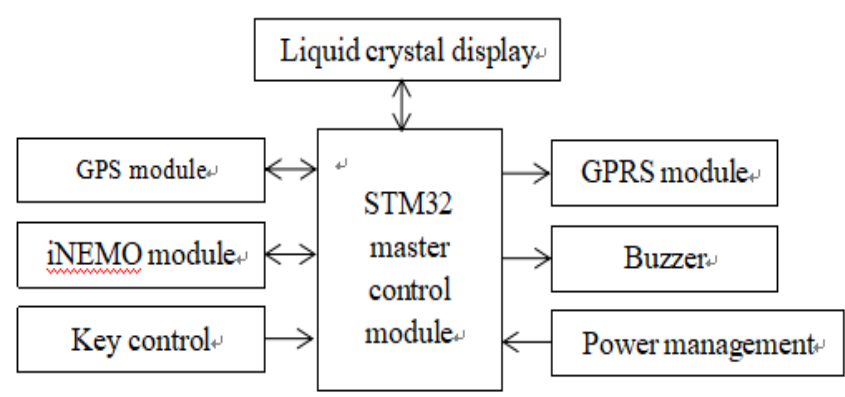

Figure 1 Overall block diagram of the system

\section{HARDWARE CIRCUIT DESIGN}

\subsection{Master Control Module Design}

The main control chip is STM32F103VCT6 of STMicroelectronics company. The design of the minimum system circuit is shown in Figure 2, including BOOT circuit, crystal oscillator circuit, reset circuit, power supply circuit, etc.

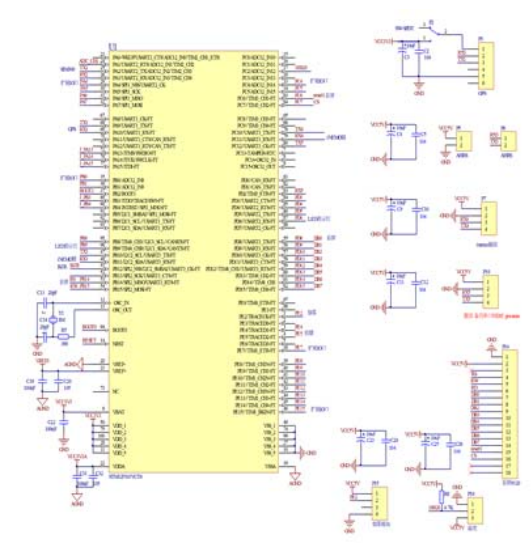

Figure 2 Minimum system diagram of STM32F103

\subsection{GPS Module Design}

This system adopts the GPS module GS-216m with ceramic antenna. Gs-216m is an intelligent satellite receiving module with high efficiency and low power consumption. GPS module continuously sends data to the main controller STM32 through the serial port.

\subsection{GPRS Module Design}

SIM300 is selected as GPRS wireless transmission module. SIM300 is designed with low power consumption, and the current consumption is only $2.5 \mathrm{~mA}$ in sleep mode; SIM300 integrates TCP / IP protocol stack and extends TCP / IP at instruction, which makes it easy for users to develop data transmission equipment by using this module. The application circuit of SIM300 chip is shown in Figure 3.

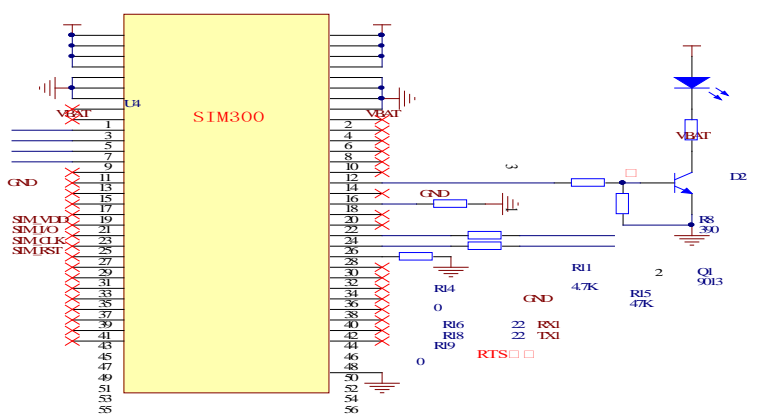

Figure 3 Circuit diagram of SIM300 
The RXD and TXD pins are adopted to conduct serial communication with the SCM STM32 and receive the data and instructions sent by the STM32. The SIM card information Reading circuit is shown in figure 4.

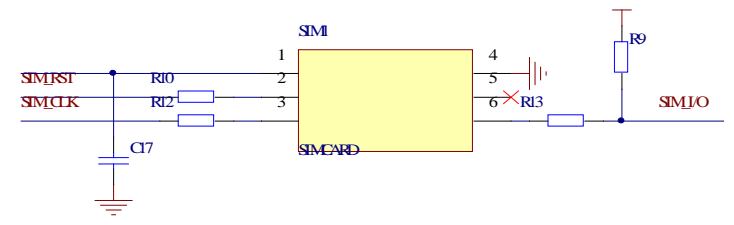

Figure 4 SIM card information Reading circuit

\subsection{Serial Communication Module Design}

The serial communication uses MAX3232 chip for level conversion. STM32 adopts serial communication with GPS, iNEMO and GPRS, STM32 uses serial port 2 to send instructions to GPRS through MAX3232 level conversion and receive the returned information; The main control board provides $3.3 \mathrm{~V}$ voltage to supply GPS power, use serial port 1 to receive GPS longitude and latitude information; Power supply voltage of leg and waist inertial navigation module is $5 \mathrm{~V}$, leg iNEMO and waist iNEMO respectively use serial port 3 and serial port 4 to receive angle and acceleration information. The MAX3232 circuit is shown in figure 5 .

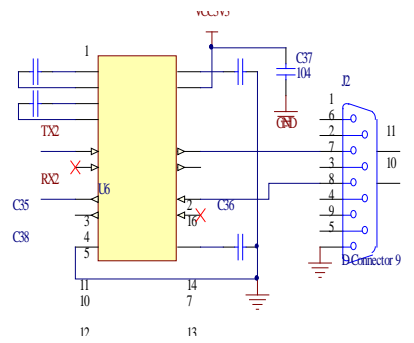

Figure 5 MAX3232 circuit

\subsection{Inertial Navigation Module Design}

The inertial navigation module adopts the iNEMO sensor produced by Italian semiconductor company. The iNEMO sensor can provide raw data through serial port, and also can provide pitch angle, rolling and yaw after AHRS algorithm fusion.

\subsection{Display Module Design}

The TFT2.4-inch color screen is selected to display the user's position, posture and other information. The TFT ILI9320 is selected as the display control part of the color screen, and the color screen interface P14 is designed. CS is the color screen selection pin, reset1 is the RESET pin, RS is the data command selection pin, and RW is the read/write selection pin. The color screen display circuit is shown in figure 6 .

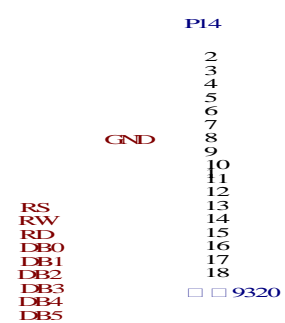

Figure 6 Color screen display circuit interface

\subsection{Power Module Design}

The system selects LM2940-5.0 voltage stabilizing module to output 5V DC voltage, and selects LM117-3.3 voltage stabilizing module to output 3.3V DC voltage. The power supply circuit is shown in Figure 7.
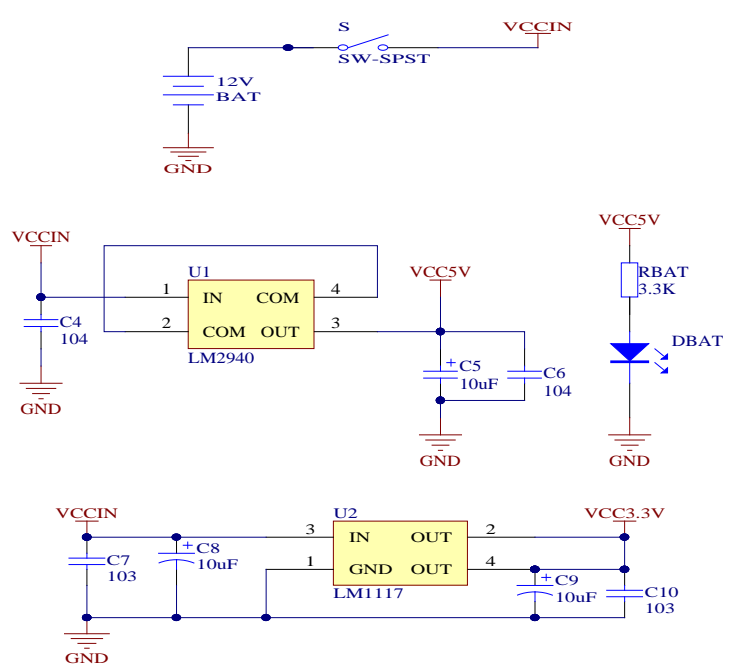

Figure 7 Power Circuit Schematic diagram 

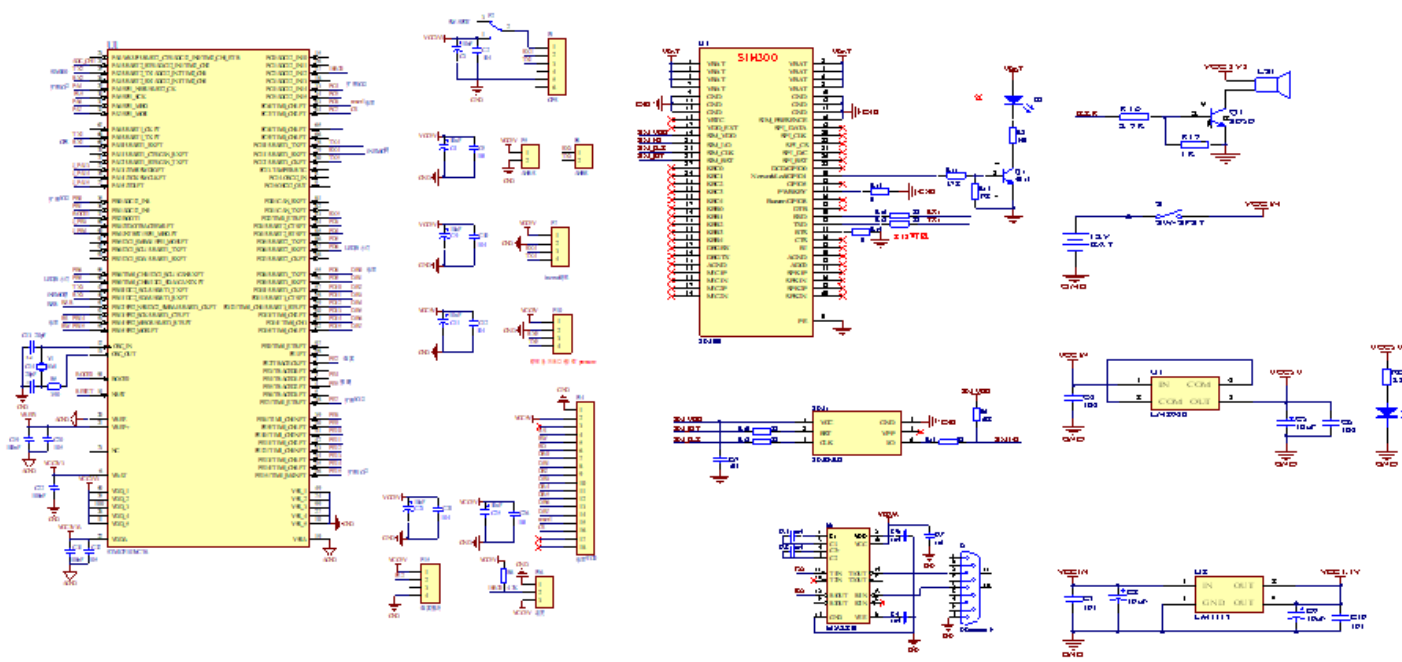

Figure 8 Circuit system structure diagram

\section{SYSTEM COMMUNICATION DESIGN}

The positioning and attitude detection system communicates with the upper computer through sockets and uploads information such as pitch angle, yaw angle and attitude to the upper computer. The specific communication mechanism is shown in Table 1. Among them, the upper computer starts to parse the data packets uploaded by the system through frame head recognition, and the 2-20 bits are the location information collected by GPS. After processing, the upper computer converts it into the required format, 21-26 is the three-axis acceleration information collected by iNEMO sensor. PDR algorithm is realized by combining the yaw angle of iNEMO sensor at waist. After analyzing the pitch angle and yaw angle of iNEMO sensor at waist and leg, realize remote positioning and attitude detection.

Table 1 Upper and lower computer communication protocol

\begin{tabular}{|c|c|c|c|c|l|l|}
\hline Function & 0x55 0xAA & GPS & ACC & YAW & PITCH & 0x66 0xBB \\
\hline Byte & $0-1$ & $2-20$ & $21-26$ & $27-28$ & $29-30$ & $31-32$ \\
\hline $\mathbf{0 - 1}$ & \multicolumn{3}{|c|}{ Frame Header } \\
\hline $\mathbf{2 - 2 0}$ & \multicolumn{3}{|c|}{ 2-11: Latitude, 11-20: longitude } \\
\hline $\mathbf{2 1 - 2 6}$ & Acceleration values of X, Y, and Z axes \\
\hline $\mathbf{2 7 - 2 8}$ & Yaw angle of lumbar inertial navigation module \\
\hline $\mathbf{2 9 - 3 0}$ & Pitch angle of leg inertial navigation module \\
\hline $\mathbf{3 1 - 3 2}$ & \multicolumn{3}{|c|}{ Frame End } \\
\hline
\end{tabular}




\section{CONCLUSION}

In order to verify the positioning and attitude detection performance of the system, the positioning and attitude detection functions were tested respectively.

\subsection{Positioning test}

The positioning result of the test is shown in Figure 9, the red point is the starting point of positioning, and the red line represents the actual motion track. The test proves that the walking track coincides with the map display of the upper computer, and the positioning function is successfully realized.

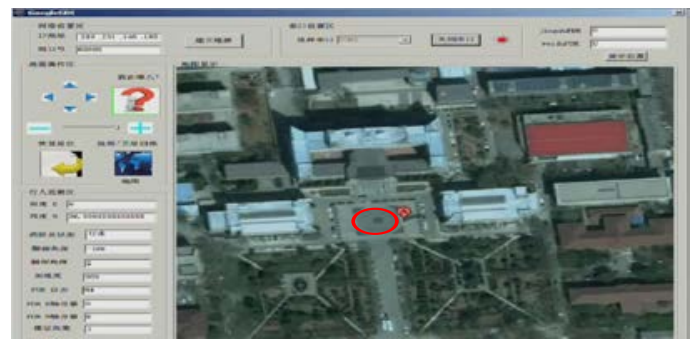

Figure 9 Positioning test results

\subsection{Attitude detection test}

The test results of personnel attitude test are shown in figure 10. The test has tested a series of continuous actions such as standing, walking, standing, bending down, sitting down, lying down, standing and lying down. The test proves that, the upper computer can accurately display the Test action and realize the attitude detection function.

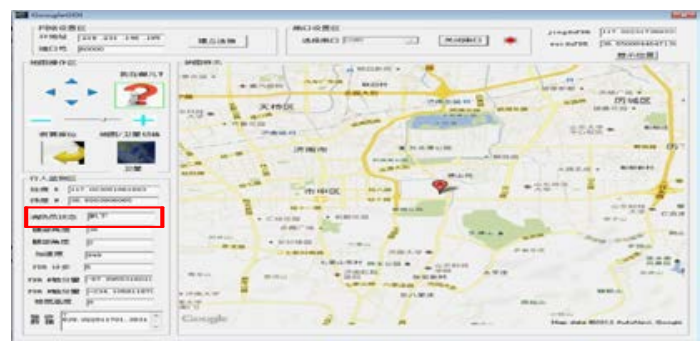

Figure 10 Attitude detection test results

\section{ACKNOWLEDGMENT}

The authors wish to thank Fengyu Zhou, Prof. for his assistance in conducting this study.

\section{REFERENCES}

[1] WANG Y H, LI L, ZHENG Z Q, etal. An improved algorithm for indoor and outdoor seamless positioning based on GPS and ultra-wide band[J].Journal of Shanghai Normal University (Natural Sciences),2021,50(1):84-91.

[2] Research on High Precision Seamless Positioning Model and Method Based on Multi-sensor Fusion[D].China University of Mining and Technology,2020.

[3] Wei Chen. Research on GPS/Self-contained Sensor Based Seamless Outdoor/Indoor Pedestrian positioning Algorithm[D].University of science and technology of China,2010.

[4] Development of Vehicle Remote Controller Based on OSEK / VDX Norm[D].Hunan University,2008.

[5] Hardware design of seamless positioning and attitude detection based on GPS and inertial navigation[D].Shandong University,2013.

[6] Han Weiji.The Applied Research of Navigation Electronic Map Based On GPS in VC++ Environment[D].Harbin Engineering University,2011.

[7] Wang Kun,Chen Xinzhi.Research on Dynamic Level of Groundwater Monitoring System Based on GPRS[J].Computer Measurement\& Control,2011.19(2):263-265.

[8] H. Li, P. Xu, et. al., Low-voltage and fast-response $\quad \mathrm{SnO} 2$ nanotubes/perovskite heterostructure photodetector, Nanotechnology, 32(2021)375202.

[9] Wang, Y., Wang, W., Yang, X., et. al., ITGA8 positive cells in the conventional outflow tissue exhibit Schlemm's canal endothelial cell properties, Life Sciences, 278(2021)119564.

[10] J. He, X. Liu,et al. High Annealing Stability of InAlZnO Nanofiber Field-Effect Transistors with Improved Morphology by $\mathrm{Al}$ Doping. Journal of Physical Chemistry Letters, 12.4(2021)1339-1345. 
[11] H. Zu, Y. Chang, H. Li, et. al., Modulating the Transport Properties of Metal Oxide Nanofibers Transistors by Controlling the Grain Size. IEEE Electron Device Letters. 42.6(2021)855-858.

[12] P. Xu, J. Cao, et. al., Quantum chemical study on the adsorption of megazol drug on the pristine BC3 nanosheet, Supramolecular Chemistry, 33(2021)63-69. 